\title{
Hysteresis and Economics
}

\section{Taking the economic past into account}

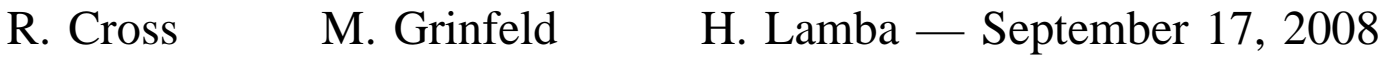

The goal of this article is to discuss the rationale underlying the application of hysteresis to economic models. In particular, we explain why many aspects of real economic systems are hysteretic is plausible. The aim is to be explicit about the difficulties encountered when trying to incorporate hysteretic effects into models that can be validated and then used as possible tools for macroeconomic control. The growing appreciation of the ways that memory effects influence the functioning of economic systems is a significant advance in economic thought and, by removing distortions that result from oversimplifying specifications of input-output relations in economics, has the potential to narrow the gap between economic modeling and economic reality.

Although economic systems evolve in time, mainstream economics on the whole is not cognizant of the history dependence of economic processes, even though the relevance of the historical dimension in economics has been stressed time and again by historians and philosophers, for example, in [1], which is also one of the first works in economics to mention hysteresis explicitly (but without providing a rigorous definition).

Merely accepting that economic structures evolve is not sufficient to single out any 
particular mechanism of history dependence at work. In many cases an economic agent can be represented by a hysteron, a simple input-output system with weak, passive hysteresis. There are three key facts implying that representing an individual economic agent as a hysteron is relevant to economic analysis. First of all, in many economic decisions the agent is confronted with a binary choice, namely, to keep savings in the local currency or in US dollars, to produce shoes or not to produce shoes, and to buy a particular stock or to sell it. Furthermore, such decisions are translated into action almost instantaneously. Finally, there are frequently sunk costs associated with switching between states. Sunk costs refer to the situation where, when an action is taken such as buying equities or entering a market to produce a commodity, an expense is incurred that cannot be recouped on reversing the action. Examples are transaction costs when buying equities and the payment of local taxes when deciding to open a production line. When sunk costs are present, a reversal of strategy is not expected to occur under exactly the same circumstances that led to the original strategy being adopted. If such circumstances can be described in terms of a single control parameter, such as the price of an equity or the production cost of a commodity, a way to model decision making in the presence of sunk costs is to postulate the existence of different threshold values of the control parameter for switching from one strategy to the other. As is shown below, this is precisely what a hysteron does.

Economic agents, such as individual consumers or firms, differ with respect to their economic preferences, ease with which they can be spurred into action, and the range of economic activities available to them. In terms of hysterons, taking the heterogeneity of agents into account corresponds to postulating different threshold values for each economic agent, and points toward Preisach-type hysteresis models as a vehicle for describing economic systems. In particular, the viewpoint described above makes it possible to understand the provenance of hysteresis loops 
in relations among macroeconomic variables, and introduces the concepts of rate independence, coercivity, and remanence into the analysis of diverse spheres of economic activity[2].

The structure of this article is as follows. First static hysteresis input-output (I/O) systems, hysterons, and Preisach models are defined, and the form that macroeconomic models with hysteresis typically take is described. Then some relevant economics background is sketched, and the distinctive nature of models in economics is discussed in detail. In the following central section of the article the results of approximately two decades of hysteresis modeling in economics are summarized. In that section we also describe in detail the thinking behind considering economic agents as input-output systems with hysteresis. Finally, stray thoughts are collected and pointers to the future in this area of research are highlighted.

\section{Systems with Hysteresis}

In this section the models to be described subsequently are placed within the general theory of systems with hysteresis. The general theory has matured significantly in the last 20 years; for details on its origins, applications, and mathematical structures the reader is referred to [3], [4], [5], [6], [7] and the economics-oriented exposition in [8].

Consider an input-output system $S$ with scalar input $u(t)$, scalar output $s(t)$, and some initial state $u(0)=u_{0}, s(0)=s_{0}$. This system $S$ is a system with memory if, at time $t$, the output $s(t)$ is determined by the input history $\{u(\tau), \tau \in(0, t]\} \subset \mathbb{R}$ and not just by $u(t)$. Therefore there is no single-valued mapping from $\mathbb{R}$ into $\mathbb{R}$ that associates each value of the current output $s(t)$ with the current input $u(t)$. Instead, such a mapping, denoted by $\mathcal{H}$, is set-valued, and, for all $t, s(t) \in \mathcal{H}(u(t))$. In order to fully describe the input/output relationship in a system with 
memory we introduce the operator $F$ defined by

$$
s(t)=F(\{u(\tau), \tau \in(0, t]\}):=F[u](t) .
$$

Note that this relationship defines a mapping between function spaces. First fix time $T>0$, and let $u$ be an element of a space $X$ of real-valued functions defined on $[0, T]$, for example $X=$ $C([0, T])$, or $X=L^{p}([0, T])$, the spaces of continuous and $p$-integrable functions respectively. Then define a function $s$ on $[0, T]$ pointwise by $s(t)=F[u](t)$. The analysis of systems with memory requires knowing the space $Y$ that $s$ belongs to, and whether or not the mapping $F: X \rightarrow Y, F(u)=s$ is continuous.

Now suppose that time is reparameterized by a mapping $t \mapsto h(t)$, where $h^{\prime}(t)>0$, and set $u_{h}(t)=u(h(t))$. If, for every reparameterization $h$ and every value of $t$, it follows that $F\left[u_{h}\right](t)=F[u](t)$, then $S$ is rate independent. For applications in economics the most appropriate definition of a hysteretic system is the following one.

Definition: The system $S$ is hysteretic if it is a rate-independent system with memory.

The quintessential example of a hysteretic system is a hysteron [3] (this object is called a relay in [5]), which is defined as follows. Let $\alpha, \beta$ be real numbers with $\alpha<\beta$, and assume that there exists a time $t^{*}$ such that

$$
t^{*}=\max \{\tau \in(0, t] \mid u(\tau)=\alpha \text { or } \beta\}
$$

where $u(t)$ is the system input. The output $s(t)$ of a hysteron is given by

$$
s(t):=F_{\alpha \beta}[u](t)= \begin{cases}1, & \text { if } u(t) \geq \beta \text { or if } u(t) \in(\alpha, \beta) \text { and } u\left(t^{*}\right)=\beta, \\ -1, & \text { if } u(t) \leq \alpha, \text { or if } u(t) \in(\alpha, \beta) \text { and } u\left(t^{*}\right)=\alpha .\end{cases}
$$

The mapping $F_{\alpha \beta}: X \rightarrow Y$ is not a continuous mapping for any choice of function spaces $X$ and $Y$. Note also that the output $s(t)$ is constrained to lie on the union of two curves in $\mathbb{R}^{2}$, 
$C_{L}=\{(x,-1), x \in(-\infty, \beta)\}$ and $C_{U}=\{(x, 1), x \in(\alpha, \infty)\}$. This observation motivates the following definition [5].

Definition: Let $S$ be a hysteretic system, and assume that the set-valued map $\mathcal{H}$ has the property that, for all $u \in \mathbb{R}$, the set $\mathcal{H}(u)$ contains a finite number of points. Then the system has weak hysteresis. Otherwise it has strong hysteresis.

Consider the hysteretic system $S$ at time $t_{0}$ with output $s_{0}=s\left(t_{0}\right)$ and input $u_{0}=u\left(t_{0}\right)$. The rate-independence property of $S$ implies that, in the absence of changes in the input, the output remains constant. Now suppose that the input $u(t)$ changes from $u_{0}$ to some value $u_{1}$ and back again. Then for each $u_{0}$ there are values $u_{1}$ such that, after the excursion, the output does not return to $s_{0}$ but instead to some different value $s_{1}$. For a hysteron in state $s\left(t_{0}\right)=-1$ and $u_{0} \in(\alpha, \beta)$, examples of such values are $u_{1} \geq \beta$ or $u_{1} \leq \alpha$. This phenomenon is known as remanence. To return the output variable to its original value $s_{0}$, we need to change the input by an additional amount, called the coercive force. These concepts are illustrated in Figure 1.

The counterclockwise dynamics of a hysteron under a periodic input is typical of a system with weak hysteresis; counterclockwise traversal of a hysteresis loop is often referred to as passive hysteresis [9]. However, there exist hysteretic elements with more complicated dynamics than that of a hysteron; toward the end of this article an example of a model of stockmarket dynamics is provided where an element flips its state whenever either threshold value is passed and the thresholds themselves change each time the state changes. Nevertheless a system of such elements is hysteretic by our definition and displays remanence. Another difference is that, for a hysteron, if a threshold value is crossed, then we only need to know the historical record of the input to predict the state of the element. However, in the model of [10] the initial 
conditions continue to influence the dynamics for all later time.

In economics applications of hysteresis it is often natural to assume that the input $u(t)$ evolves in discrete time $t_{k} \in \mathbb{Z}$. Thus, if $u\left(t_{k}\right), s\left(t_{k}\right)$ are given and $u\left(t_{k+1}\right)$ is prescribed next, we interpolate $u\left(t_{k}\right)$ and $u\left(t_{k+1}\right)$ by a monotone continuous function $\widehat{u}(t)$, where $t \in\left[t_{k}, t_{k+1}\right)$, $\widehat{u}\left(t_{k}\right)=u\left(t_{k}\right), \widehat{u}\left(t_{k+1}\right)=u\left(t_{k+1}\right)$, and $s(t)=F[\widehat{u}](t), t \in\left[t_{k}, t_{k+1}\right)$.

Many economic systems can be represented by large assemblages of heterogeneous elements each having simple dynamics, for example, that of a hysteron. However, it is not clear whether an assemblage of hysteretic elements under a common input $u(t)$ is itself a hysteretic system with some appropriately defined aggregate output variable. A useful class of strong (passive) hysteresis systems arises when we set

$$
s(t)=\mathcal{P}[u](t):=\int_{\Gamma} g(\alpha, \beta) F_{\alpha \beta}[u](t) d \alpha d \beta,
$$

where $g(\alpha, \beta)$ is a continuous function with support in $\Gamma \subset\left\{(\alpha, \beta) \in \mathbb{R}^{2} \mid \beta \geq \alpha\right\}$, such that

$$
\int_{\Gamma} g(\alpha, \beta) d \alpha d \beta=1
$$

and $\operatorname{Area}(\Gamma) \neq 0$. Such systems are called Preisach systems and the function $g(\alpha, \beta)$ is the Preisach weight (density) function. The standard reference for the theory, applications, and generalizations of such models is [11]. Preisach hysteresis operators $\mathcal{P}[u]$ have good continuity and monotonicity properties [6], [5], which makes systems with Preisach hysteresis operators especially amenable to analysis. The dynamic features of Preisach systems, such as the remanence property discussed above, as well as the wiping-out and congruence properties, are described by the Mayergoyz staircase construction [12], [11], [8]. In brief, a hysteretic system has the wiping-out property if the output is uniquely determined by the sequence of non-dominated 
extrema of the input; and a system with strong hysteresis has the congruence property if all interior loops generated by cyclical excursions of input between two values, say $u_{0}$ and $u_{1}$, $u_{0}<u_{1}$, are geometrically congruent (see, for example, [12, Figure 2]). Systems with Preisach hysteresis operators in discrete time are considered in [13].

A Preisach system exhibits hysteresis at both the micro and the macro level. However, many models of macroscopic hysteretic systems do not require hysteretic units at the micro level. Perhaps the best-known class of such models is the random field Ising model of Barkhausen noise [14], [15]; for a recent economics application see [16]. The key to obtaining hysteresis at the macroscopic level is to assume enough heterogeneity among the components in the form of thresholds chosen at random from some distribution with appropriate coupling. More precisely, if the system consists of $N$ components with the $i$-th component at time $t_{k}$ having state $s^{i}\left(t_{k}\right) \in$ $\{0,1\}$, the zero-temperature random field Ising dynamics [14] update rule is given by

$$
s^{i}\left(t_{k+1}\right)=\operatorname{sgn}\left[\sum_{j=1}^{N} J_{i j} s^{j}\left(t_{k}\right)+f^{i}+u\left(t_{k}\right)\right],
$$

where $f^{i}$ are the random thresholds and $J_{i j}$ are the coupling parameters.

Note that the hysterons in a Preisach system are uncoupled. Each hysteron reacts only to the input $u(t)$ and there is no feedback mechanism allowing a change in the state of a hysteron to affect $u(t)$. However, assuming that the units are decoupled is too restrictive in economics. Hence an appropriate class of models in economics is one in which units are hysteretic, time is discrete, and units are coupled. Assume a Preisach hysteresis operator; such a system can be written

$$
\begin{aligned}
& u\left(t_{k+1}\right)=u\left(t_{k}, s\left(t_{k}\right), \ldots\right) \\
& s\left(t_{k+1}\right)=\mathcal{P}[u]\left(t_{k+1}\right),
\end{aligned}
$$


where ellipses may stand, for example, for exogenous information entering the system. A model of this type is used in [17] to describe market entry-exit decisions of firms.

\section{Economics background}

The foundations of the mainstream model of contemporary economics were laid in the neoclassical revolution of the 1870 s. In contrast to the preceding classical economics, where the analysis focused on capitalists, workers and landlords considered as collective entities, the starting point in neoclassical economics is the individual economic agent. Households consume goods and accumulate assets through savings and supply labor inputs into production. Firms produce goods, use savings to finance production, and have a demand for the labor and capital inputs used in production. These economic agents are characterized as being predominantly selfinterested. Households maximize utility functions that depend upon the goods they consume and the labor services they supply, subject to budget constraints matching expenditure and income. Firms maximize their profits, determined by revenues minus costs, subject to production function constraints describing feasible production techniques. From this postulated behavior, individual demand and supply schedules are derived, which depend on the prices of the consumer goods and production inputs. In the simplest setup, households and firms are price takers, with the agents regarding the prices as given outside of their control. Individual demand and supply schedules are then aggregated to form market demand and supply functions, with functional dependence on prices.

The key question is then whether there is a set of prices that can reconcile the interests of buyers and sellers so that aggregate quantities demanded equal aggregate quantities supplied on 
all markets. This question was initially addressed by invoking a deus ex machina, in the form of an unpaid auctioneer, who would announce a set of prices, record the excesses of demand over supply, and converge to a market-clearing price vector by adjusting prices upward or downward in markets with excess demand or supply, respectively. This unsatisfactory solution, proposed by the former-engineer Walras in 1874 [18], relies on the number of unknown prices being at least matched by the number of equations in the form of excess demand functions. In contrast, modern Arrow-Debreu proofs of the existence of such a market-clearing price vector use fixed point theorems and rely on assumptions such as convexity in consumption and production sets [19].

The pioneers of neoclassical economics tended to have a mathematics, physics, or engineering background and relied heavily on metaphors drawn from Newtonian mechanics when constructing their theories of value, that is, the determination of relative prices [20]. Market equilibrium was thus seen as a balance of forces. The responses of quantities to changes in prices were defined in terms of elasticities, and, in Fisher's account, the individual agent was seen as a particle, the commodity was seen as a type of space, marginal utility corresponded to force, disutility to work, and utility to energy [21, p. 85]. Indeed a central place in Fisher's Ph.D. thesis is taken by a hydrostatic model of water flowing through pipes to interconnected cisterns. This model illustrates how the marginal utility of consumption and the marginal cost of production are brought into balance at the market equilibrium price. The key characteristic of this theory of value is that equilibrium market prices reflect the marginal utility of the marginal consumer and the marginal cost of the marginal producer. Figure 2 reproduces Fisher's diagrammatic representation of his model [21, p. 56]. 
From the 1930s neoclassical economics was reformulated on an axiomatic basis, but the mathematical techniques used in the general equilibrium existence and stability proofs preserved the properties imported by the metaphors of Newtonian mechanics [20], [19]. In particular, conservation of energy was retained in the economic context to imply that nothing is lost or permanently changed if an individual, a market, or the economy as a whole face a temporary disturbance, no matter how large. For example, during the US Civil War, the burning of the cotton fields reduced the supply of cotton. In the neoclassical account this disturbance would have only temporary effects, the market for cotton returning to the status quo ante after the war. Utility functions describing tastes and production functions describing production possibilities would be unchanged, and the replanting of the cotton fields post bellum would be accompanied by a return to the original equilibrium. One of the great expositors of neoclassical economics, Marshall [22, pp. 425-426, p. 660, p. 667], saw the limitations of this framework. Tastes for non-cotton goods acquired during the disturbance would not be forgotten, nor would productivity gains arising from learning-by-producing in non-cotton goods industries be lost. The result would be a form of hysteresis, the temporary disturbance having lasting effects mediated by way of changes in tastes and production relationships. For related arguments and evidence, see [1], [23].

\section{Macroeconomics}

The main concerns of macroeconomics are with the determination of aggregate output, unemployment rate, and inflation. We now describe briefly the standard account of the dynamics of these indicators of economic activity. 
Aggregate output is measured in national accounts using definitions such as gross domestic product (GDP). Relations link instantaneous national output, income, and expenditure, once adjustments are made for international trade, government expenditure, and taxation. Say's Law, that supply creates its own demand, summarizes the neoclassical wisdom that there cannot be a shortage of demand provided that the forces underlying supply and demand are not fettered by government interference. The value added in production is distributed as income to the factors of production. Income is spent on consumed goods or saved. Savings are channeled into satisfying the investment demand for new capital goods, where the rate of interest moves to eliminate any discrepancy between the demand for and supply of loanable funds. In this view, business cycle downswings are associated with disturbances such as bad harvests — caused by sunspot activity variations in one account - but are temporary since the losses in output are recovered in the subsequent upswing phase.

The term unemployment came into usage in the English language only in the 1880s. Any lack of employment tended originally to be attributed to physical or moral deficiencies in those without work and not to coordination failures in economic systems. In the natural rate of unemployment theories of neoclassical economics [24], the equilibrium, or natural, rate of unemployment, which is consistent with a steady rate of inflation, depends largely on the degree of government intervention in the form, inter alia, of minimum wages, state unemployment benefits, and trades union or labor-market regulations. Such interventions raise real wages above the market-clearing levels. Thus, according to natural rate theory, if unemployment is perceived to be too high, the solution is to dismantle government interventions in labor and other markets.

Inflation is measured as the rate of change of some index of prices, the main headline 
figure being that for consumer prices. The traditional quantity theory of money explains the rate of inflation by the prior change in the quantity of money. In the early 20th century the quantity theory was formalized as $M V=P T$ by Fisher, where $M$ is the nominal stock of money, $V$ is the velocity of its circulation, $P$ is the level of the price index, and $T$ is the flow of real transactions or the aggregate output flow. The velocity $V$ is taken to be fixed by institutional arrangements such as cheque clearing, and $T$ by the conditions of aggregate supply. Hence the dichotomy between the theory of value and that of the price level. The causation is assumed to be in the direction $M \rightarrow P$, ignoring the possibility of reverse causation. For more information on central bank attempts to control inflation, see "Inflation and Taylor rules".

Keynes presented the most influential challenge to the orthodoxy of neoclassical economics. He answered his question "Is the Economic System Self-Adjusting?" in the negative [25], arguing that free markets would not necessarily generate full employment, which would be the natural rate of employment in the current terminology. The problem, as he saw it, was that private-sector consumption and investment plans are formed in the face of expectations about the future economic environment that are inherently uncertain and subject to shifts that have more to do with emotions than with the rational calculations postulated in neoclassical economics. As a result, shortfalls of effective demand in relation to the level required for full employment occur. Hence governments have a role to play by increasing their spending, cutting taxes or reducing interest rates to make up for any deficiencies in private sector demand. The Keynesian revolution stimulated the work of Phillips [26]; see "Phillips and his Machine" for details.

If a market-based economic system does not necessarily self-adjust to full employment, as Keynes argued, then there is a control problem. Tinbergen, the first director of the Dutch 
Central Planning Bureau in 1945, posed the control problem in the context of fixed reference values for macroeconomic targets such as output, employment and the balance of payments[27]. Econometric estimates of macroeconomic relationships are used to describe the impact of policy input variables, such as government spending and interest rates, on the output variables. A key issue is whether there would be a sufficient number of policy instruments to allow the simultaneous achievement of the reference values of the policy targets. There are many problems with the Tinbergen approach to macroeconomic control, including imperfections in the data describing the current and past states of the economy, uncertainty about the best econometric description of the economy, uncertainty about the values of policy instruments set by foreign policymakers, and arbitrariness in the choice of reference target values. Subsequent approaches attempted to deal with these problems by framing the control problem in terms of a policymaker maximizing a preference function defined over policy objectives (the national equivalent of an individual agent's utility function) subject to constraints describing the way the economy is perceived to function.

The above approach to macroeconomic control has been out of fashion since the 1970s. The policy ineffectiveness critique associated with the natural rate hypothesis [24] claims that macroeconomic policy instruments or input controls do not have lasting effects on real policy targets such as unemployment and real GDP. Macroeconomic policies are claimed to have lasting effects only on nominal variables such as the rate of inflation, hence the switch of attention to how inflation policy targets can be achieved by Taylor-type rules [28]. The associated Lucas critique [29] argues that private-sector agents have rational expectations that take into account all the relevant information contained in a model of their behavior when forming their (usually differing) expectations. Thus their behavior is not invariant with regard to the policy interventions 
of governments or their agencies. A related tendency is to argue that the preference function of the policymaker should somehow reflect the preferences of individual economic agents, with policy outcomes reflecting a dynamic game played between policymakers and individual economic agents. The incorporation of hysteresis into the analysis of economic systems can not only provide new analytical foundations for Keynes' views on the existence of equilibria at less than full employment, but also resurrect the control problem denied by the policy ineffectiveness proposition of neoclassical economics.

\section{The Methodology of Macroeconomic Modeling}

As noted above, the mainstream model of neoclassical economics relies heavily on metaphors drawn from classical physics, Newtonian mechanics in particular, and in doing so imported conservation and reversibility principles into its analysis of economic systems. The obvious question to ask is whether these properties are observed in the workings of real economic systems. Alternative approaches to economic analysis tend to rely on metaphors drawn from contexts in which conservation and reversibility do not hold. For example, evolutionary economics takes evolutionary biology as its metaphor source, while the present article considers hysteresis, a term coined by Ewing to describe the behavior of electromagnetic fields in ferrous metals, as its source [30]. These multiple possibilities raise methodological issues such as the importance of evidential criteria and the feasibility of experiments in economics (for details, see "Experimental Economics"). It is not clear how models in economics can be validated and whether they can be used for prediction and control.

Some of the changes that occur in economic systems can be considered as naturally 
occurring experiments, albeit ones lacking a carefully controlled reference group. Therefore researchers can look to see whether economies recover to their previous trend growth paths for output after a recession, or merely return to the previous level [31]. Alternatively, researchers can ask whether the move to inflation-targeting by independent central banks is accompanied by a reduction in the size of real GDP or unemployment fluctuations. In both these examples the Duhem-Quine thesis raises problems, since it is not clear by what criteria the obtained results confirm the hypothesis under test or leave doubts as to whether other factors, such as institutional changes or globalization, might explain the findings.

The main vehicle for testing hypotheses in economics is econometrics, which is concerned with drawing inferences from non-experimental time series. Data on variables deemed to be exogenous or endogenous are used to attempt to identify structural economic relationships, or conduct tests on the nature of the reduced form relationships. A basic problem is that the error terms can reflect a wide set of phenomena, such as measurement errors in the variables, excluded variables, and model mis-specification as well as any inherent noise or nonstationarity in the underlying processes. Despite the waves of optimism that have accompanied the unveiling of new econometric techniques, the results have not yielded what might be described as highly robust empirical findings. Some hypotheses can be dismissed as unlikely because they are data-incoherent. However, even apparently data-coherent relationships rarely generate consensus amongst economists. Trawling large data sets for statistically significant correlations, in the absence of a specific underlying hypothesis to test, is understandably regarded with suspicion by theoretical economists - especially by those of an opposing school of economic thought.

The problems involved in testing theories in economics help to explain why many 
economists place so much emphasis on consistency with underlying principles (such as utilityfunction maximization by economic agents) when choosing between rival theories. Evidence does play a part, but more in the way of qualitative properties, such as whether economic processes display mean reversion and drift back to their long-term average after an economic shock, or follow a random walk. A discussion on this topic can be found in [32].

On at least two key points the hysteresis account of economic systems is promising. Firstly, neoclassical economics tends to simplify or ignore the aggregation problem by assuming the existence of representative economic agents, whose optimizing decisions are scaled up to represent the behavior of all consumers or producers. This assumption is inconsistent with the observation that individuals differ, and ignores the implications of agent heterogeneity (see [33] for a corrosive account). The ability of Preisach models to incorporate agent heterogeneity in their hysteron representations is certainly an improvement in this respect.

The second point relates to the issue of where consumer preferences or production techniques come from. In the neoclassical account, tastes and technologies are taken as exogenous to the economic system. This approach ignores the obvious presence of learning-by-doing in consumption and production.

\section{Hysteresis Models in Economics and Finance}

The rôle of hysteresis in economic and financial modeling is now considered. Most such models are based on representing individual economic agents as hysterons, an approach that provides, as argued below, an attractive characterization of various microeconomic scenarios such as the entry and exit of firms in a particular market. The hysterons can then be aggregated to 
provide a macroeconomic model of total output and employment. Note that due to the possible feedback mechanisms between agents' states and the inputs (for example, increased supply reducing the price) the aggregated system is not strictly a Preisach model but rather is of the form (1).

As outlined above, much of neoclassical economics is predicated on the existence of a unique, stable, history-independent equilibrium. Based on this paradigm, the central banks of many countries, but interestingly not of the US, are restricted to using monetary policy to try and achieve a target rate of inflation. Yet remanence and coercivity in economic activity at the macroeconomic level are observed [31], [34], and these effects of monetary policy have to be taken into account. We thus contrast the mainstream model in finance, that of efficient markets, with models where hysteresis effects are present.

\section{Economics}

We first ask under what circumstances an economic agent can be adequately represented by a hysteron. Such a representation is predicated on there being a binary choice on the part of the agent, with the associated switching involving sunk costs. The magnitudes of sunk costs are a major factor in determining the threshold values $\alpha<\beta$ for a given agent and the ensuing heterogeneity of the model population. Secondly, the switching time of the hysteron must be fast compared to the timescale of the model and the variations in the input $u(t)$. If at some point the input stops changing, the current states of the agents are maintained for a significant time; in other words, there is a negligible amount of switching.

These assumptions hold, for example, in the following situation. Consider a simple case 
where the relative price of capital in terms of output is normalized to unity, so that one unit of capital is used to produce one unit of output. In the standard neoclassical account of investment decisions each firm estimates an internal rate of return (IRR) on each possible investment project. The IRR is the rate of return that sets the discounted value of future revenues net of operating costs equal to the projects' capital costs. The IRR is then compared to the cost of capital funds, which can be written as a markup factor $\lambda(t)>1$ on the short-term interest rate $i(t)$ set by the central bank; this short-term interest rate is the repo rate, the rate at which the central bank lends money to private banks by repurchasing qualifying assets.

If investment projects were costlessly reversible, in that the capital costs of a project could be fully recouped if the project were abandoned, the knife-edge conditions IRR $>\lambda(t) i(t)$ or IRR $<\lambda(t) i(t)$ would determine whether or not the firm would or would not go ahead with the project in the first place, or continue with or abandon the project if the latter is already under way. The evidence, however, is that firms require rates of return substantially in excess of the cost of capital funds, typically three or four times the cost of capital [35], before they proceed with investment projects. A highly plausible explanation for this phenomenon is that capital projects involve sunk costs that are not recoverable if the project is abandoned. If an oil exploration project is abandoned in the face of a fall in the price of oil, the second-hand price of the drilling rig is likely to be at a substantial discount to the purchase price; the sales, distribution network, and advertising costs of bringing a new product to the market would be lost if the product flops; and so on. This account provides the rationale for reformulating the condition for the capital project to proceed as $[\operatorname{IRR}-\lambda(t) i(t)] \geq \beta$, where $\beta$ is the upper trigger in a hysteron. Once a capital project has been begun the decision of the firm is whether or not to keep the project active. Because of the sunk costs, and also because of economic uncertainty 
regarding future net revenues, which depend inter alia on the expected future price of oil in the drilling rig example, and on fashion in the case of a new product, the firm will not abandon the project until a significantly lower trigger $[\operatorname{IRR}-\lambda(t) i(t)] \leq \alpha$ is reached.

Similar microeconomic foundations for representing economic agents as hysterons in Preisach-based models are provided in [8] for the cases of how exports and imports respond to exchange-rate changes, how the hiring and firing of workers is related to sudden changes to aggregate demand, and how output in the member countries of the European Monetary union responds to the interest rates set by the European Central Bank [2]. The crucial element describing how economic agents respond to input variables is the presence of sunk costs in the adjustment of economic behavior. This conclusion matches the observation that in many contexts economic adjustments are made relatively infrequently, and in large doses, rather than responding more or less continuously to even small changes in input variable, as neoclassical models imply.

The qualitative properties of Preisach-based economic models are analyzed in [36] using the general results on systems with hysteresis obtained in [3], [11], in particular the staircase partition representation of the division between active and inactive hysterons. These models plausibly suggest that economic systems contain a selective, erasable memory of the nondominated extrema of perturbations to input variables. In terms of business cycles this property means that major recessions and booms leave permanent effects in their wake, rather than merely representing temporary deviations from some given growth path, as in the neoclassical account. The implications of Preisach-based models for business cycles are considered in [37]. Methods for analyzing how recessions leave curses in their wake in the form of a lower growth-path for outputs, and how booms can leave blessings, in the form of a higher growth-path, are presented 
in [38] and [39], providing a framework for incorporating Preisach memory-effects into the analysis of macroeconomic systems in general. Little work is available on the determination of the rate of inflation in hysteretic systems. An account of output-inflation interaction is needed to fill this gap, requiring a shift toward vector hysteresis models.

A major problem in conducting empirical tests on Preisach-based models is the lack of information on the Preisach weight function $g(\alpha, \beta)$. In the example of capital investment projects considered above there is the inherent problem that the trigger values $\alpha$ and $\beta$ are regarded by firms as commercially sensitive information. In other cases there is also a dearth of cross-sectional data on the switching points that allow economic agents to be represented as hysterons. There is also the problem that the switching points could well change over time as agents learn from mistakes or otherwise change their strategies for responding to the economic environment[40].

Two empirical studies illustrate both the promise and difficulties associated with testing Preisach-based models in economics. In [34] such a model is used to investigate how hysteresis affected the equilibrium rate of employment in the UK, 1959-1996. The key finding is that hysteresis index variables reflect a selective memory of exchange rates, oil prices and interest rate perturbations. To generate the hysteresis index variables, the area under the Mayergoyz staircase partition [11] is approximated as a union of rectangular trapezoids. The Preisach weight function is first specified as a uniform distribution and then sensitivity tests are conducted using normal, Poisson, and exponential distributions. These tests suggest that the alternative distributional assumptions make little difference to the results. A limitation is that the time-series variables contain few peaks and troughs, thus making the results tentative. Higher frequency data in 
relation to financial markets might permit firmer conclusions to be drawn. In [41] the empirical problem is to explain the way US imports from Japan respond to changes in the dollar-yen exchange rate. The strategy here involves piecewise-linear approximations of macro-hysteresis loops, where the slope of the linear functions change at extrema. Again the results are positive in that the hysteresis effect is found to be statistically significant, but the empirical method could be at best described as an approximation. To date there is little work on the control problems arising in such Preisach models in economics.

It is unfortunate that the most common usage of the term "hysteresis" in economics differs significantly from that defined in this article or employed in the physical sciences. This term is used by many economists to refer in a very general way to the persistence of deviations from equilibrium, especially after severe economic shocks [42], [43]. The mainstream explanation of persistence of deviations runs as follows. Suppose that the system can be considered as a linear, discrete-time, stochastic difference equation of the form

$$
X_{t}=A X_{t-1}+\eta_{t}, \quad X_{t} \in \mathbb{R}^{m}
$$

where $\left|\eta_{t}\right| \ll 1$ is an exogenous stochastic process and $A \in \mathbb{R}^{m \times m}$. Further, assume that all the eigenvalues of $A$ lie inside the open unit disk so that the origin is an asymptotically stable equilibrium, and let $a$ be the eigenvalue of largest magnitude. If an economic shock moves the system away from equilibrium then long transients can be generated if $a \approx 1$, which leads to a history-dependent system path over a long time. There is a vast literature on the existence of unit root processes with econometric tests claiming to have detected their presence [44], [45].

The above phenomenological model begs some basic questions. Firstly, it is not clear that there is any a priori reason why economic equilibria should have an eigenvalue close 
to the boundary of the unit disk. This being the case, the worrying possibility rises that economic systems are inherently borderline unstable, with profound implications for the rest of macroeconomics. Secondly, if memory effects are in fact due to the presence of hysteresis and remanence, and not to the presence of unit roots, how does this affect the statistical tests (for example, the augmented Dickey-Fuller test [46]) for unit roots?

To summarize, suggesting that hysteresis is indeed occurring at microeconomic levels, makes it possible to employ the phenomenon of selective memory and remanence to provide a plausible, and relatively well understood, explanation for persistence and path dependence in macroeconomics. Furthermore, hysteresis provides a mechanism by which history dependence and stability can comfortably coexist, in direct contrast to the unit root hypothesis.

\section{Financial Markets}

The Efficient Market Hypothesis

The consequences, both philosophical and practical, of the assumptions underlying the hypothesis of memory-free efficient financial markets cannot be overestimated. Although the concepts were introduced by Bachelier in his 1900 Ph.D. thesis, this work was largely forgotten until the 1960s when the concepts became known collectively as the efficient market hypothesis $(\mathrm{EMH})$ [47], [48], [49]. Firstly, there are strong assumptions about the market itself and the nature of the information stream entering it. These data consist of economic statistics, performance reports, geopolitical events, and analysts' projections. It is assumed to be instantly available to all economic participants, uncorrelated with itself, and is usually modeled as a Brownian 
motion, possibly with drift. A second class of assumptions relates to the market participants themselves, who are deemed to be perfectly rational and capable of instantaneously incorporating new data into their differing market strategies and predictions. The heterogeneity of agents is necessary to ensure that trading occurs in the absence of arbitrage opportunities (arbitrageurs are agents who can identify and act upon instantaneous riskless profit opportunities due to small market mispricings). Thus the final ingredient in the EMH description is the rational expectations assumption that the differing expectations driving trades, when used as predictions, are on average correct and do not result in market mispricing. Additional assumptions, such as the absence of transaction costs, yield the standard formulae used for risk management and derivative pricing which form the bedrock of modern financial "engineering".

Numerous statistical studies of actual markets and asset prices show significant deviations from the implications of the EMH [50], [51]. These differences, which are surprisingly independent of geography, asset type, trading rules and political systems, are known as the stylized facts. We now briefly discuss the two most well-known types of deviations. Volatility clustering, also known as heteroskedasticity, is the phenomenon whereby the volatility of a financial variable, such as an asset price, varies over time. Volatility clustering is often quantified by measuring the autocorrelation function of the absolute value of the price returns, which decays slowly over several months according to an approximate power law. However, the autocorrelation function of the price returns becomes negligible over a time-scale of several minutes, in almost perfect accordance with the EMH. The typical distribution of the observed price returns (as opposed to the well-behaved linear autocorrelation) provides a second major discrepancy. Under the idealized EMH assumptions this distribution is log-normal, that is, the logarithm of the price returns over some constant interval, measured in days, weeks or months is Gaussian and thus 
has exponentially decaying tails. In fact, the tails decay much more slowly, again obeying an approximate power law, and so the standard EMH models underestimate the frequency of large price changes by many orders of magnitude. These fat tails associated with large price changes are often the manifestation of asset price bubbles or the ensuing crashes.

Models assuming constant volatility and log-normal price changes are routinely used to perform risk analysis and to price financial derivatives, in the celebrated Black-Scholes [52] option pricing formula, for example, despite firm evidence to the contrary. It is also interesting to note that the volume of financial derivatives transactions exploded when the option pricing formula was published in 1973. Prior to this, options were traded only very thinly, and usually as direct contracts between parties rather than being sold openly via an exchange. The publication

of a formula involving only one free parameter, the price volatility, provided the necessary reassurance that such derivatives could be traded as widely as the underlying asset. However, even today, most participants in the derivatives market do not fully appreciate the importance and unreliability of the underlying assumptions. This interplay between models and the behavior of economic agents adds to the points made earlier about the unusual rôle that models play in economics and finance compared with other disciplines.

\section{Modeling markets with hysteretic agents}

An immediate consequence of the EMH is that markets have no memory. In other words, all past information is accurately and instantaneously incorporated into the current stock price so that nothing is to be gained by looking at past market data. The sometimes schizophrenic nature of economics and finance is indicated by the fact that this notion of 'the market is always 
right' is upheld by many of the same people who hire technical analysts or chartists to pore over past data to predict future price moves. A possible counterargument is that such technical analysis itself forms part of the pricing mechanism and helps make markets more efficient than they would otherwise be.

We now show how an EMH model with rational expectations involving hysteretic agents can be constructed [53]. Hysteretic agents by definition are history dependent, while the EMH models, by definition, are not. However, the rational expectations framework allows individual agents to have memory dependence provided that, when averaged, this dependence does not manifest itself in the asset price. Thus consider a highly simplified system of $M$ agents, each of whom is able to be long (own) or short (not own) only one unit of an asset. The discrete-time system evolves in time steps of length $h$, and the investment position of the $i^{\text {th }}$ investor over the $n^{\text {th }}$ time interval is represented by $s_{i}(n)= \pm 1(+1$ own, -1 not own $)$. The price of the asset at time $n$, which is denoted by $p(n)$, is subject to an exogenous information stream in the form of a Brownian motion $W(n)$, where the time variable $t$ is scaled so that the variance of $W(t)$ over a unit time interval is 1 . We define sentiment as the average of the states of all of the $M$ investors given by

$$
\sigma(n)=\frac{1}{M} \sum_{i=1}^{M} s_{i}(n)
$$

Further defining $\Delta \sigma(n)=\sigma(n)-\sigma(n-1)$, the price is updated using the formula

$$
p(n+1)=p(n) \exp (\sqrt{h} \eta(n)-h / 2+\kappa \Delta \sigma(n))
$$

where $\kappa \geq 0$ and $\sqrt{h} \Delta W(n) \sim \mathcal{N}(0, h)$ represents the exogenous information stream. If $\kappa=0$ then the price follows a geometric (driftless) Brownian motion determined only by the external information stream. But when $\kappa>0$ the price now also depends on internal dynamics by means 
of the market sentiment term reflecting the changing investment positions of the agents.

Each agent is modeled in terms of binary switches. Suppose that at time $n$ the $i^{\text {th }}$ investor switches and the current price is $P$. Then a pair of numbers $X_{L}, X_{U}>0$ is generated from some specified distribution (independent of the particular agent), and the lower and upper price thresholds for that agent are set to be $L_{i}=P /\left(1+X_{L}\right)$ and $U_{i}=\left(1+X_{U}\right) P$, respectively. The agent is considered to switch instantaneously when either $p(n)>U_{i}$ or $p(n)<L_{i}$. When such an event occurs, a new set of thresholds straddling the current price is generated. Now suppose that $M$ is large and $\sigma(0) \approx 0$, with the initial states of the agents well mixed. Then the lack of any coupling between agents implies that over any time step the numbers of agents switching in each direction cancels and $\sigma$ remains close to 0 . Thus, the behavior of the system is close to the case where $\kappa=0$ and EMH pricing still applies. Further details, economic justifications and numerical simulations can be found in [54], [55], [56], [53].

The above model matches the rational expectations and EMH paradigm, that is, that agents trade because of differing future expectations but the price remains correct because there is no coupling between agents and the differences cancel. However, the threshold values are capable of multiple economic interpretations in addition to the neoclassical one of rational economic analysis. Firstly, the presence of thresholds naturally incorporates the effects of transaction/sunk costs, exactly as described above for entry-exit problems. Secondly, the psychological pressure to take profits or cut losses (depending on which threshold is breached) is captured by the hysteron description. Also, experimental economists and psychologists have demonstrated the existence of anchoring, where investment decisions are strongly influenced by recent experience, in this case the last price at which the asset was traded by that agent. There is now a substantial literature 
categorizing and attempting to quantify such psychological propensities [57], [58], [59], [60], [61], [62], [63], [64].

As simple as the above model is, it already differs significantly from the standard use of Preisach hysterons in, say, ferromagnetics. In the continuum limit $M \rightarrow \infty$, the state of all agents at time $n$ can be represented by a weight function, which is analogous to the Preisach weight function but evolves in time, loosely shadowing the price $p(n)$ as agents switch to keep the price within their thresholds. This dynamic property, which can be contrasted with the static Preisach weight function of a magnetic material, hints at the much greater potential for complex behavior in financial markets than in magnets. This complexity reveals itself when coupling between the agents is introduced. Then the EMH pricing property of the model can be lost, as is now shown.

In [53], building on previous work [54], [55], [56], a herding tendency is introduced into the agents' behavior. The phenomenon of herding is well documented and appears to be a contributing factor in most, if not all, financial bubbles. There are several underlying reasons for herding to occur. Firstly, there is the psychological propensity for people to feel safer when in the majority, and the positive feedback in the form of momentum trading can mean that yet more people take the same position. Secondly, there are significant (rational but perverse) reasons why professional investors herd into similar market positions. These individuals or their institutions often cannot afford noticeably to underperform the market even for short periods without losing their jobs or investment capital. In [53] the herding effect is modeled by allowing the hysteron thresholds to move between switchings, the thresholds move inwards towards the current price 
whenever that agent is in the minority, following the rule

$$
L_{i}(n+1)=L_{i}(n)+C_{i} h|\sigma(n)|, \quad U_{i}(n+1)=U_{i}(n)-C_{i} h|\sigma(n)|,
$$

where the $C_{i}$ are agent-dependent parameters reflect the agent's herding propensity. This change means that agents in the minority are now more likely to switch into the majority position than vice versa. The effect of this change is that significant asset mispricings occur, with fat-tailed price returns similar to those observed in real markets. The herding effect does not however induce volatility clustering, which is then introduced by additionally supposing that the volume of high-frequency or "noise" trading is correlated with the market sentiment $\sigma$. Note that this ability to infer causal relationships between EMH-violations at the micro-level and non-EMH statistics at the macro-level (at least within this modeling paradigm) arises precisely because the hysteron approach provides a framework within which the EMH assumptions can be replicated and then systematically weakened.

The output of random processes fed through a Preisach filter is studied in [65], [66]. The above model suggests that financial markets can potentially be viewed the same way, albeit with the added complication that the weight function is itself evolving over time. The dynamics and control of such coupled hysteretic-unit systems provides a formidable but fascinating challenge.

\section{Conclusions and Outlook}

This article has considered the use that economists have made of hysteresis concepts, borrowed originally from micro-magnetics and adapted to various contexts of economic activity. Though highly suggestive and intuitively attractive, these concepts have so far had at best an informal influence on economic policy. Their main use has been to frame criticisms of mainstream 
models that either do not take into account any history dependence or consider only the special case of unit roots in the underlying difference equations. In order that hysteresis, in addition to suggesting an explanatory mechanism, becomes a formal tool in the policy makers armory, much work remains to be done.

From the theoretical point of view, it is desirable to characterize those interactions among micro-hysteretic economic agents that do, and those that do not, lead to macroscopic hysteresis. Another outstanding issue is the rigorous derivation of mean-field models in systems with hysteretic microstructure [54]. A challenging empirical task is to ascertain experimentally, in various decision-making contexts, to what extent the behavior of actual economic is hysteretic. Properties of agents should be studied both in isolation, when the decisions of an agent do not impinge on the economic data that she has to respond to, and in interaction with other agents, in order to understand the types of information used in economic decision-making and the nature of the interaction. Detailed surveys are also required to identify the switching points involved in the Preisach weight functions, and how these evolve. Such foundations are required to construct robust models of economic systems with hysteresis, which can then be used to address the control problems that can arise.

\section{Acknowledgments}

We are grateful to Mrs. I. Spencer for her help in the preparation of the manuscript. 


\section{References}

[1] N. Georgescu-Roegen, The Entropy Law and the Economic Process. Cambridge, Mass.: Harvard University Press, 1971.

[2] M. Göcke, "Various concepts of hysteresis applied in economics," J. Econ. Surveys, vol. 16, pp. 167-188, 2002.

[3] M. A. Krasnosel'skii and A. V. Pokrovskii, Systems with Hysteresis. New York: SpringerVerlag, 1989.

[4] A. Visintin, Differential Models of Hysteresis. New York: Springer-Verlag, 1994.

[5] J. W. Macki, P. Nisri, and P. Zecca, "Mathematical models for hysteresis," SIAM Review, vol. 35, pp. 94-123, 1993.

[6] M. Brokate, "Hysteresis operators," in Phase Transitions and Hysteresis, A. Visintin, Ed. Heidelberg: Springer-Verlag, 1994, pp. 1-38.

[7] O. Hovorka, "Hysteresis behaviour patterns in complex systems," August 2007, Ph.D. Thesis, Drexel University. [Online]. Available: http://idea.library.drexel.edu/bitstream/1860/ 1791/1/Hovorka_Ondrej.pdf

[8] T.-A. Borgersen and M. Göcke, "Exchange-rate overshooting and path-dependence in international trade," Macroeconomic Dynamics, vol. 11, pp. 295-317, 2007.

[9] J. C. Hsu and A. U. Meyer, Modern Control Principles and Applications. New York: McGraw-Hill, 1968.

[10] R. Cross, M. Grinfeld, H. Lamba, and T. Seaman, "A threshold model of investor psychology,” Physica A, vol. 354, pp. 463-478, 2005.

[11] I. D. Mayergoyz, Mathematical Models of Hysteresis. New York: Academic Press, 2003. 
[12] _ _ "Mathematical models of hysteresis," Phys. Rev. Lett., vol. 56, pp. 1518-1521, 1986.

[13] S. A. Belbas and I. D. Mayergoyz, "Optimal control of dynamical systems with Preisach hysteresis," Int. J. Non-linear Mech., vol. 37, pp. 1351-1361, 2002.

[14] J. P. Sethna, K. Dahmen, S. Kartha, J. A. Krumhansl, B. W. Roberts, and J. D. Shore, "Hysteresis and hierarchies: dynamics of disorder-driven first-order phase transitions," Phys. Rev. Lett., vol. 70, pp. 3347-3350, 1993.

[15] J. P. Sethna, K. A. Dahmen, and O. Perković, "Random-field Ising models of hysteresis," in The Science of Hysteresis, Vo. II, G. Bertotti and I. D. Mayergoyz, Eds. Amsterdam: Academic Press, 2006, pp. 107-179.

[16] C. Borghesi and J.-P. Bouchaud, "Of songs and men: a model for multiple choice with herding," Qual. Quant., vol. 41, pp. 557-568, 2007.

[17] L. Piscitelli, M. Grinfeld, H. Lamba, and R. Cross, "Exit-entry decisions in response to aggregate shocks," Appl. Econ. Lett., vol. 6, pp. 569-572, 1999.

[18] L. Walras, Elements of Pure Economics (1874). London: Allen and Unwin, 1954, translated by W. Jaffee.

[19] E. R. Weintraub, General Economic Analysis: Studies in Approval. New York: Cambridge University Press, 1985.

[20] P. Mirowski, More Heat Than Light. New York: Cambridge University Press, 1979.

[21] I. Fisher, Mathematical Investigations in the Theory of Value and Prices. New Haven, CT.: Yale University Press, 1925.

[22] A. Marshall, Principles of Economics. London: Macmillan, 1920, 8th ed., 1st ed. 1890.

[23] G. Calvo, A. Izquierdo, and E. Talvi, "Phoenix miracles in emerging markets," March 2006, nBER Working Paper No.12101, http://www.nber.org/papers/w1210. 
[24] M. Friedman, "The role of monetary policy," American Economic Review, vol. 58, pp. $1-17,1968$.

[25] D. Moggridge, Ed., The Collected Writings of John Maynard Keynes, Vol. XIII. Macmillan/ Cambridge University Press, London, 1973.

[26] A. W. H. Phillips, "Mechanical models in economic dynamics," Economica, vol. 18, pp. $283-305,1950$.

[27] M. L. Petit, Control Theory and Dynamic Games in Economic Analysis. New York: Cambridge University Press, 1991.

[28] J. B. Taylor, "Discretion versus rules in practice," Carnegie Rochester Series on Public Policy, vol. 39, pp. 195-214, 1993.

[29] R. E. Lucas, "Econometric policy evaluation: a critique," Journal of Monetary Economics Supplement, vol. 1, pp. 19-46, 1976.

[30] J. A. Ewing, "On the production of transient electric currents in iron and steel conductors by twisting them when magnetised or by magnetising them when twisted," Proceedings of the Royal Society of London, vol. 33, pp. 21-23, 1881.

[31] V. Cerra and C. S. Saxena, "Growth dynamics: the myth of economic recovery," Bank for International Settlements, Tech. Rep. 226, March 2007, http://www.bis.org/cbhub/sourcebiswp.htm.

[32] A. Rubinstein, Modeling Bounded Rationality. Cambridge MA: MIT Press, 1998.

[33] A. Kirman, "Who or what does the representative agent represent?" Journal of Economic Perspectives, vol. 6, pp. 117-136, 1992.

[34] J. Darby, R. Cross, and L. Piscitelli, "Hysteresis and employment: a preliminary investigation," in The Science of Hysteresis I, G. Bertotti and I. Mayergoyz, Eds. Amsterdam: 
Academic Press, 2006, pp. 667-699.

[35] A. Dixit, "Investment and hysteresis," J. Econ. Persp., vol. 6, pp. 107-132, 1992.

[36] R. Cross, "Hysteresis and emu," Metroeconomica, vol. 51, no. 4, pp. 367-379, 2000.

[37] H. McNamara and A. K. Pokrovskii, "Hysteresis in the trade cycle," Physica B, vol. 372, pp. 202-206, 2008.

[38] R. Cross, H. McNamara, A. Pokrovskii, and D. Rachinskii, “A new paradigm for measuring hysteresis in economic flows," Physica B, vol. 403, pp. 231-236, 2008.

[39] R. Cross, H. McNamara, and A. K. Pokrovskii, "Modelling macroeconomic flows related to large ensembles of elementary exchange operations," Physica B, vol. 403, pp. 451-455, 2008.

[40] R. Cross, A. M. Krasnosel'skii, and A. K. Pokrovskii, "A time-dependent Preisach model," Physica B, vol. 306, pp. 206-210, 2001.

[41] M. Göcke, "Approximation of the hysteresis loop by linear partial functions - econometric modelling and estimation," Jahrbücher für Nationalökonomie und Statistik, vol. 213, pp. 572-596, 1994.

[42] F. Giavazzi and C. Wyplosz, "The zero root problem," Review of Economic Studies, vol. 52, pp. 353-357, 1985.

[43] B. Amable, J. Henry, L. J., and R. Topol, "Hysteresis revisited: a methodological approach," in The natural rate of unemployment, R. Cross, Ed. Cambdridge: Cambridge University Press, 1995.

[44] A. Bannerjee, J. Dolado, J. W. Galbraith, and D. F. Hendry, Co-Integration, Error Correction, and the Econometric Analysis of Non-Stationary Data. Oxford UP, 1993.

[45] K. Patterson, Unit Roots in Economic Time Series. Palgrave Macmillan, forthcoming, 
2009.

[46] E. Said and D. Dickey, "Testing for unit roots in autoregressive moving average models of unknown order,' Biometrica, vol. 71, pp. 599-607, 1984.

[47] E. Fama, "The behavior of stock market prices," Journal of Business, vol. 38, pp. 34-105, 1965.

[48] P. Samuelson, "Proof that properly anticipated prices fluctuate randomly," Industrial Management Review, vol. 6, pp. 41-49, 1965.

[49] E. Fama, "Efficient capital markets: A review of theory and empirical work," J. Finance, vol. 25 , pp. $383-417,1970$.

[50] R. Mantegna and H. Stanley, An Introduction to Econophysics. Cambridge, UK: CUP, 2000.

[51] R. Cont, "Empirical properties of asset returns: stylized facts and statistical issues," Quantitive Finance, vol. 1, pp. 223-236, 2001.

[52] F. Black and M. Scholes, "The pricing of options and corporate liabilities," J. Polit. Economy, vol. 81, pp. 637-659, 1973.

[53] H. Lamba and T. Seaman, "Rational expectations, psychology and inductive learning via moving thresholds," Physica A, vol. 387, pp. 3904-3909, 2008.

[54] R. Cross, M. Grinfeld, and H. Lamba, "A mean-field model of investor behaviour," J. Phys.: Conference Series, vol. 55, pp. 55-62, 2006.

[55] H. Lamba and T. Seaman, "Market statistics of a psychology-based heterogeneous agent model," preprint, To appear, Int. J. Theor. Appl. Fin.

[56] R. Cross, M. Grinfeld, H. Lamba, and T. Seaman, "Stylized facts from a threshold-based heterogeneous agent model," Eur. J. Phys. B, vol. 57, pp. 213-218, 2007. 
[57] D. Kahneman and A. Tversky, "Judgement under uncertainty: heuristics and biases," Science, vol. 185, no. 4157, pp. 1124-1131, 1974.

[58] D. Kahneman, P. Slovic, and A. Tversky, Judgement Under Uncertainty: Heuristics and Biases. Cambridge University Press, 1982.

[59] R. H. Day and V. L. Smith, Experiments in Decision, Organization and Exchange. Amsterdam: North-Holland, 1993.

[60] J. H. Kagel and A. E. Roth, Eds., The handbook of experimental economics. Princeton, N. J.: Princeton University Press, 1995.

[61] H. Simon, Models of Bounded Rationality. Cambridge, Mass.: MIT Press, 1997.

[62] R. Thaler, "From homo economicus to homo sapiens," J. Econ. Perspectives, vol. 14, pp. 133-141, 2000.

[63] D. Kahneman, "A psychological perspective on economics," Amer. Econ. Rev., vol. 93, pp. 162-168, 2003.

[64] N. Barberis and R. Thaler, "A survey of behavioral finance," in Handbook of the Economics of Finance, G. Constantinidos, M. Harris, and R. Stultz, Eds. Amsterdam: Elsevier Science, 2003, pp. 1053-1123.

[65] C. Korman and I. Mayergoyz, "Switching as an exit problem," IEEE Trans. Magn., vol. 31, pp. 3545-3547, 1995.

[66] —_ "Preisach model driven by stochastic inputs as a model for aftereffect," IEEE Trans. Magn., vol. 32, pp. 4204-4209, 1996.

[67] P. Krugman, "Who was Milton Friedman?" The New York Review of Books, vol. 54.2, 15 February 2007.

[68] C. Bissell, "The Moniac: a hydromechanical analog computer of the 1950s," IEEE Control 
Systems Magazine, vol. 27, pp. 59-64, 2007.

[69] R. Leeson, Ed., A. H. W. Phillips: collected works in contemporary perspective. Cambridge: Cambridge University Press, 2000.

[70] S. D. Levitt and J. A. List, "What do laboratory experiments measuring social preferences reveal (about the real world)?” J. Economic Perspectives, vol. 21, pp. 153-174, 2007.

[71] F. Guala, The Methodology of Experimental Economics. Cambridge: Cambridge University Press, 2005. 


\section{Sidebar 1: Inflation and Taylor Rules}

In a metallic-currency world, the supply of money for monetary use is determined by gold or silver mining or extraction rates, less the demand for the metals in non-monetary use. Paper money was exchangeable for gold under the Gold Standard, so the total money supply tended to follow that of gold. This policy imparted stability to the price level, the British price level at the start of the 1914-18 World War, for example, being the same as it was fifty years previously. The Bretton Woods international monetary system of 1944-71 involved pegging currencies to the US dollar, which in turn was exchangeable for gold at $\$ 35$ per ounce. Once the last links to gold were abandoned, attempts to implement the monetarist prescription of controlling inflation by having central banks operate non-feedback rules for the rate of change of the money supply were tried in various countries. By and large these attempts failed since central banks were unable to hit their monetary targets in a world of deregulated financial markets [67]. Since the late 1980s the typical monetary control regime has come to be one of independent central banks pursuing inflation targets. This system relies on the natural rate hypothesis that $\dot{p}=f\left(u-u^{*}\right)+\dot{p}^{e}$, where $\dot{p}$ and $\dot{p}^{e}$ are the actual and expected rates of inflation, and $u$ and $u^{*}$ are the actual and natural rates of unemployment. For $\dot{p}=\dot{p}^{e}$, the conditions $u=u^{*}$ and $f(0)=0$ need to hold. Therefore, for inflation expectations to be consistent with the target rate $\dot{p}^{*}$ of inflation, central banks need to respond to any emerging discrepancies between $u$ and $u^{*}$. The Taylor rule

$$
i=a+b\left(\dot{p}-\dot{p}^{*}\right)+c\left(u^{*}-u\right)
$$

was used to describe how a central bank fixes its input repo rate control variable $i$, the interest rate at which the bank repurchases qualifying securities from banks in return for cash, in order to hit a $\dot{p}^{*}$ inflation target [28], with the feedback variables being represented by $u^{*}-u$. 


\section{Sidebar 2: Phillips and his Machine}

Phillips was an electrical engineer who became an economist after his incarceration in a Japanese POW camp, and built an analog hydromechanical machine to illustrate the workings of Keynesian macroeconomics; see [68] and Figure S1. Phillips went on to write key papers on

control, focusing on the problems raised by time lags in the responses of macroeconomic policy targets to variations in input control variables. The innovation here was the introduction of PID (proportional, integral, derivative) feedback methods to try to design a macroeconomic control system that could correct for shortfalls of GDP in relation to reference values without amplifying the cyclical fluctuations in GDP. In this work Phillips employed a relationship between the rate of inflation and the level of GDP, a forerunner of the estimated curve for which he became famous [69]. 


\section{Sidebar 3: Experimental Economics}

A common misconception is that experiments are impossible in economics. There is actually an extensive literature on experimental economics going back to the 1950s, and a currently active research agenda in neuroeconomics that exploits findings from neuroscience. The 2002 Nobel prize in economics was given to the psychologist Kahneman and the economist Smith for their work on experimental economics. What is different in economics is that the experiments are artificial in the sense that the economics laboratory is populated by people who are asked to reveal the choices they would make in hypothetical circumstances that are removed from real-world economic circumstances. This situation obviously raises the question of the extent to which behavior revealed in the economics laboratory can be translated into situations in the real world, an issue being whether the incentives that can be offered in the laboratory can mimic what drives real-world economic behavior [70]. At least some of the laboratory-derived results have proved to be robust predictors of actual behavior, in auctions, for example. Kahneman's experimental results include those identifying loss aversion, reference dependence and anchoring in the heuristics used to make choices between uncertain prospects [57], [58]. Smith's work [59] is concerned, inter alia, to see whether experimental markets display key neoclassical properties, such as the efficient markets hypothesis claim that market prices reflect all the available information relevant to price determination (see [71] for an illuminating discussion of experimental economics). The Duhem-Quine thesis is particularly relevant here in that laboratory experiments require controlling for a large number of auxiliary hypotheses in order to expose a target hypothesis for refutation. 


\section{Author Information}

Rod Cross was educated at the London School of Economics and the University of York. He has held posts at Manchester, Queen Mary College, London, St Andrews, and Strathclyde, where he is currently Emeritus Professor of Economics. His main research area is in analyzing economic behavior and systems in terms of hysteresis, with other interests in transition economies, globalization, methodology, and monetary economics. He has been on the HM Treasury Academic Panel, worked as a consultant to the National Bank of Poland, and was a visiting professor at the University of Aix-Marseille.

Contact details: Department of Economics, University of Strathclyde, Sir William Duncan Building, 130 Rottenrow, Glasgow G4 0GE, Scotland, UK.

tel: 0044141548 3855/4555

fax: 00441415484445

E-mail: rod.crossestrath.ac.uk

Michael Grinfeld was educated at Tel-Aviv University, the Weizmann Institute of Science, and Rensselaer Polytechnic Institute. He is currently a senior secturer in mathematics at the University of Strathclyde. His main research area is phase transitions in material science.

Harbir Lamba was educated at Cambridge University and the University of Bristol. He is currently an associate professor in mathematics at George Mason University. His research interests include nonsmooth dynamical systems, systems with hysteresis, the numerical solution 
of differential equations, and agent-based modeling in economics. 


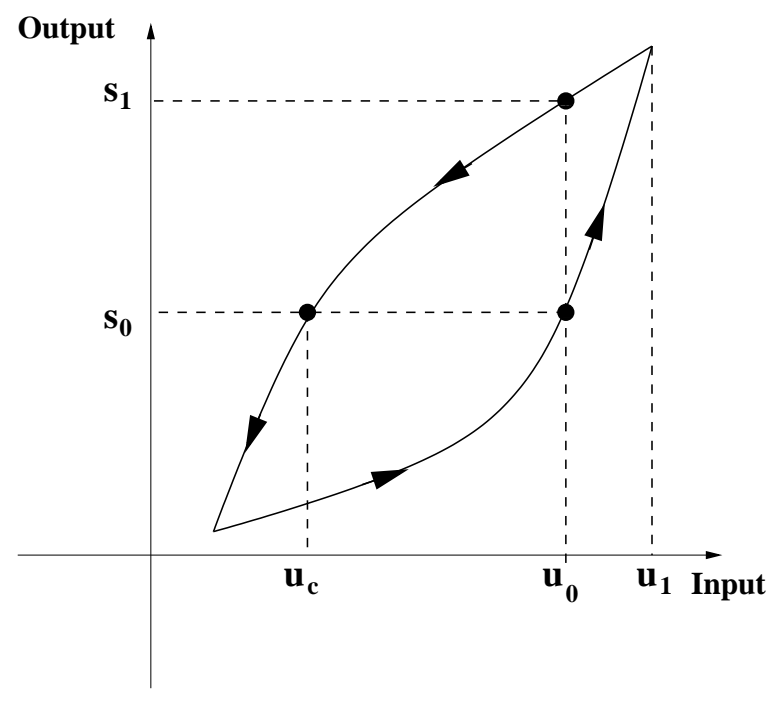

Figure 1. A hysteresis loop, remanence, and coercive force. Here the input increases to $u_{1}$ and returns to the value $u_{0}$; the remanence is the difference $s_{1}-s_{0}$, while the coercive force is $u_{0}-u_{c}$. 


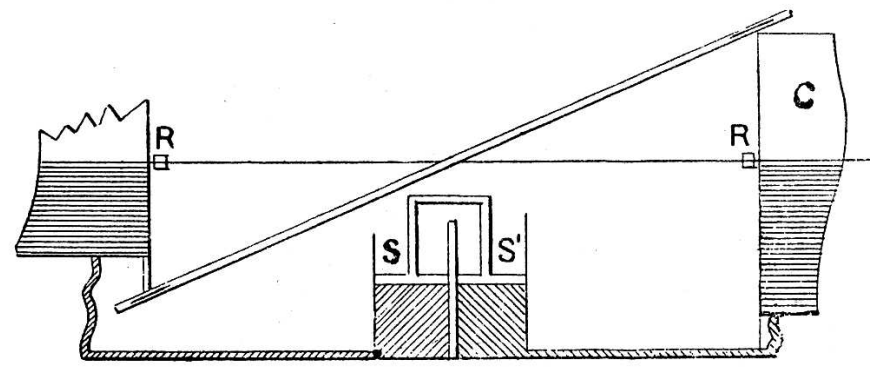

Figure 2. A diagram of Fisher's Model. The symbol C stands for cisterns associated with production and consumption that are to be found at the far left and right of the diagram, respectively. The shapes of the cisterns depict the relationships between the quantity produced and the marginal cost of production, and between the quantity consumed and the marginal utility of consumption. The symbol $\mathrm{R}$ denotes that the fixed point of the lever is placed midway between the cisterns to ensure that marginal cost equals marginal utility. The two stoppers $\mathrm{S}$ and $S^{\prime}$ regulate the quantities of liquid so that the quantities in the production and consumption cisterns are equal. The condition that supply equals demand is met by way of the duplicate pistons attached to the lever. 


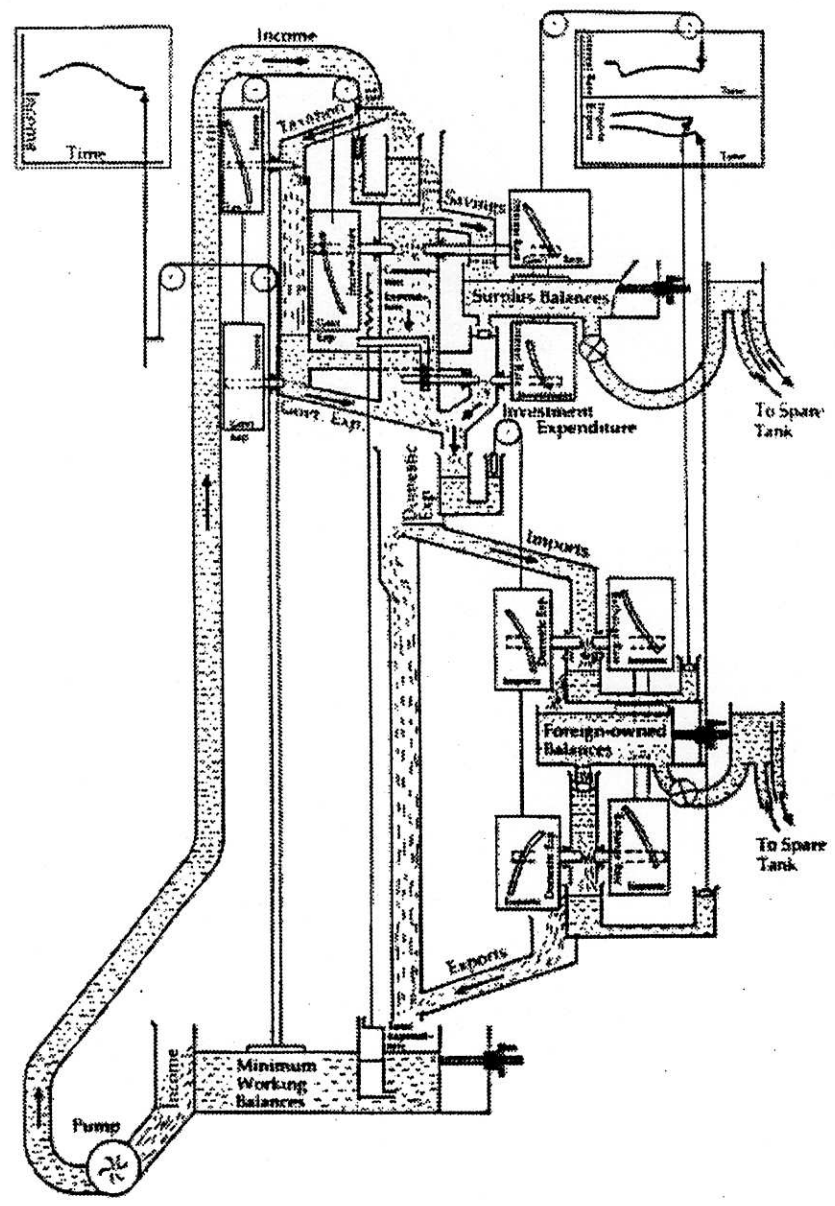

Figure S1. Phillip's machine. This device represents the macroeconomic stocks and flows by colored water flowing through tubes, with mechanical coupling through valves providing feedback from the various parts of the system. A more detailed explanation of the hydraulic and economic principles involved can be found in [68]. 